\title{
EFECTOS DEL ABONAMIENTO NITROGENADO SOBRE LA ROYA AMARILLA (Hemileia vastatrix Berck et. Br.) EN DOS VARIEDADES DE Coffea arabica L.
}

\author{
Kadir MÁRQUEZ-DÁVILA', Luis ARÉVALO¹ y Raúl GONZALES ${ }^{1}$ \\ 1 Instituto de Investigaciones de la Amazonía Peruana (IIAP). Programa de Investigación en Manejo Integral de Bosques y \\ Servicios Ambientales (PROBOSQUES).San Martín, Perú. E-mail: kmarquez@iiap.org.pe
}

\section{RESUMEN}

El presente estudio fue realizado con la finalidad de determinar los efectos del abonamiento nitrogenado sobre la roya amarilla (Hemileia vastatrix Berck et. Br.), causante de daños severos en los estadios iniciales de dos variedades de Coffea arabica L. Para ello se evaluaron cuatro dosis de Nitrógeno (N): 50, 75, 100 y 125 $\mathrm{Kgha}^{-1}$, comparadas con un control absoluto. Los tratamientos con Nitrógeno recibieron dosis constantes de fósforo, potasio, magnesio y azufre. Se registró información sobre el daño ocasionado por la roya amarilla en los estadios iniciales de las variedades de café Caturra y Borbón, durante un periodo total de doce meses. Con los datos obtenidos se calculó el porcentaje del índice de intensidad de daño (\%IID), lo que permitió determinar la curva de progreso de la enfermedad. El área bajo la curva de progreso de la enfermedad o AUDPC, se calculó con él \%IID. Con los datos de AUDPC se realizó el análisis de variancia y la prueba de Duncan para la comparación múltiple de medias $(\mathrm{P}=0.05)$. Los resultados indican que no hubo un efecto significativo y diferenciado del tratamiento en la variedad, ni tampoco hubo un efecto significativo de las dosis de Nitrogeno sobre el \%IID. Sin embargo, el abonamiento redujo significativamente él \%IID de la RA, en los estadios iniciales de ambas variedades.

PALABRAS CLAVE: Roya amarilla, Hemileia vastatrix, café, Coffea arabica, Abonamiento.

\section{EFFECTS OF NITROGEN COMPOSTING ON YELLOW RUST (Hemileia vastatrix Berck et. Br.)}

\section{ABSTRACT}

This study was developed with the objective of determining the effects of Nitrogen fertilization on severity of coffee rust (Hemileia vastatrix Berck et. Br.) (CR), in the early stages of development of two varieties of Coffea arabica L. Four doses Nitrogen $(\mathrm{N})$ : 50, 75, 100 y $125 \mathrm{Kgha}^{-1}$ were evaluated, and compared with absolute control. The treatments with $\mathrm{N}$ received constant doses of phosphorus, potassium, magnesium and sulfur. For twelve months corresponding to the initial stages the development of the Caturra and Bourbon varieties, the degree of damage of CR were recorded, with these data was calculed the percentage of the damage intensity index (\%IID), With it was determined the disease progress curve. The area under disease progress curve (AUDPC) was calculated with \%IID data. With AUDPC data, the analysis of variance and Duncan's test for multiple comparison of means $(\mathrm{P}=0.05)$ was performed. The results indicate no significant effect either of the coffee rust severity or $\mathrm{N}$ dose on the \%IID, however fertilization reduced significantly the \%IID of CR, in the early stages of development the both varieties.

KEYWORDS: Coffee rust, Hemileia vastatrix, coffea, Coffea arabica, Soilfertilization. 


\section{INTRODUCCIÓN}

El café (Coffea arabica L.) es una planta que se cultiva aproximadamente en 80 países tropicales $\mathrm{y}$ subtropicales del mundo y constituye uno de los productos agrícolas más importantes en el comercio internacional. Una mínima reducción en el rendimiento de la planta o un ligero aumento en los costos de producción por efecto de la roya amarilla (Hemileia vastatrix Berck et. Br.) (RA), puede tener un gran impacto en los caficultores de los países cuyas economías se basan, en un alto porcentaje, en las exportaciones del café (APS, 2011).

En el Perú, el café es uno de los principales productos agrícolas de exportación, generando aproximadamente el 30\% de las divisas del sector agropecuario y destinando el $95 \%$ de la producción nacional cafetalera a mercados externos. El café se cultiva en los valles interandinos de la selva alta, donde predominan los cultivares arábicos (Típica $70 \%$, Caturra 20\% y otras como Borbón y Pache $10 \%)$. El $90 \%$ de las plantas de café se cultivan bajo sombra, $75 \%$ de la producción se concentra por encima de los $1200 \mathrm{msnm}$, con un rendimiento promedio de $14 \mathrm{qq} / \mathrm{ha}$.

El rendimiento en el cultivo de café se ve afectado por los daños ocasionados por la roya amarilla. La roya amarilla es una enfermedad altamente destructiva que afecta de manera global a las economías basadas en el cultivo del café. La roya amarilla ha sido clasificada como una de las siete pestes y enfermedades más importantes durante los últimos 100 años (Agrios, 1997). La roya amarilla provoca la caída prematura de las hojas, propiciando la reducción de la capacidad fotosintética, así como el debilitamiento de árboles enfermos. En infecciones severas puede ocasionar muerte regresiva en ramas e incluso la muerte de los árboles (APS, 2011; Lara, 2005). El daño ocasionado por la roya amarilla no es inmediato, es gradual y se localiza en las hojas, pudiendo reducir considerablemente, en unos pocos años, la producción de las plantaciones, si no se ejecutan acciones de vigilancia epidemiológica fitosanitaria para determinar la severidad de daño, implementando acciones de manejo oportunas de la enfermedad (Rivillas et al, 2011; Orozco et al, 2011). Uno de los casos más severos del ataque de esta enfermedad se reportó en Ceilán (actualmente Sri Lanka), entre los años 1871 y 1878, reduciendo el área establecida para este cultivo de 68,787 a 14,170 ha, abandonándose definitivamente las plantaciones el año 1890, debido a la baja rentabilidad de las mismas (CABI, 2007).

La roya amarilla ingresa al Perú el año 1979, ocasionando grandes pérdidas en la zona de Satipo.
Los efectos de la enfermedad activaron la respuesta del estado peruano, iniciándose las acciones para el control de la enfermedad por intermedio del Ministerio de Agricultura (MINAG), basando exclusivamente el control en la utilización de productos químicos derivados del azufre, pero con deficientes conocimientos de las características del patógeno y las medidas cuarentenarias a poner en marcha (Tirado, 2008).

Durante los primeros meses del 2013, los caficultores sufrieron un ataque similar al de 1979 . E1 MINAG estimó que 500 mil personas distribuidas en 120 distritos rurales cafetaleros, fueron afectados y 60 mil productores perdieron su cosecha y vieron afectados sus ingresos durante el 2013. Todo esto generó inestabilidad social y política, así como el incremento de la pobreza y pobreza extrema (Estimados iniciales en el 2013 Junta Nacional del Café 2013). El 3 de mayo del 2013 el MINAG declara en emergencia el sector cafetalero ante el avance de la roya en el país, habilitando un presupuesto de S/. 100 millones de soles para el control de la enfermedad a nivel nacional. Al mismo tiempo, se elabora un plan de control de emergencia, con mucho énfasis en el control y uso de fertilizantes químicos y un reducido uso de fertilizantes orgánicos para las parcelas.

El estudio sobre el efecto del abonamiento nitrogenado sobre la roya amarilla en los estadios iniciales de las variedades de $C$. arabica Caturra y Borbón, permitirá elaborar planes de abonamiento adecuados para contrarrestar sus efectos, así como para combatir otras enfermedades relacionadas a las deficiencias nutricionales de las plantas. Las dos variedades de café, protagonistas del presente estudio, son de suma importancia económica, social y ambiental para la Región.

El objetivo del estudio fue determinar los efectos del abonamiento nitrogenado sobre la roya amarilla (Hemileia vastatrix Berck et. Br.), en los estadios iniciales de dos variedades de Coffea arabica L.

\section{MATERIALES Y MÉTODOS}

El experimento fue realizado en la parcela $\mathrm{El}$ Organero, de propiedad del Sr. Abraham Piña Linares, ubicada en el sector La Primavera, Distrito de Alonzo de Alvarado Roque, provincia de Lamas, región San Martín. Geográficamente la parcela está ubicada a 6 $6^{\circ} 0^{\prime} 503$ " de latitud sur, 7647'839" de longitud este y $1,411 \mathrm{msnm}$. El área experimental fue una parcela de café en proceso de renovación. 


\section{Variables en estudio}

\section{Variedades de C. arabica}

Se evaluaron dos variedades de $C$. arabica (Caturra roja y Borbón), ambas variedades con buenos antecedentes de calidad en taza y muy productivas, con una susceptibilidad elevada a la roya amarilla. Caturra es una variedad originaria de Brasil, mutación de Borbón, de porte bajo, compacto, entrenudo corto, con una excelente producción, resistente al viento. Borbón es una variedad de porte alto, entrenudos más cortos que la variedad Típica, producción elevada y con muy poca resistencia al viento.

\section{Dosis de nitrógeno}

Se evaluaron cuatro dosis de Nitrógeno (N): 50 , 75, 100 y $125 \mathrm{Kgha}^{-1}$, utilizando como fuente el nitrato de amonio ( $\mathrm{N} 33 \%$ y $\mathrm{P}_{2} \mathrm{O}_{5} 3 \%$ ). Las dosis fueron comparadas con un control absoluto (Tabla 1). Todos los tratamientos recibieron dosis constantes de: $80 \mathrm{Kg}$ de fósforo (P), $150 \mathrm{Kg}$ de potasio $(\mathrm{K}), 88 \mathrm{Kg}$ de Magnesio $(\mathrm{Mg})$ y $60 \mathrm{Kg}$ de azufre (S). Como fuente de $\mathrm{P}$ se empleó roca fosfórica $\left(\mathrm{P}_{2} \mathrm{O}_{5} 30 \%\right.$ y Ca $28 \%$ ), como fuente de $\mathrm{K}, \mathrm{S}$ y $\mathrm{Mg}$ se empleó sulfato de potasio y magnesio (K soluble $22 \%$, S soluble $22 \%$ y $\mathrm{Mg}$ soluble $18 \%$ ) (Pereira et al, 1996).

Los fertilizantes químicos fueron aplicados en dos fracciones, la primera fracción se aplicó dos meses después de la siembra a campo definitivo (mds), la segunda fracción seis meses después de la siembra. La dosis total de roca fosfórica fue aplicada a los tres meses y medio después de la siembra, abriendo un surco con forma de media luna en la parte superior de la pendiente del suelo, recubriéndolo con una capa fina de suelo y restos de tejido. Los fertilizantes fueron aplicados al voleo y cubiertos con una capa de suelo.

Los tratamientos estudiados fueron distribuidos bajo un diseño de bloques completamente randomizados (BCA), con arreglo factorial $2 \times 5$ (Variedad y Tratamientos), con diez tratamientos y 3 repeticiones.

\section{Producción de plántulas y manejo}

Para la producción de plántulas se siguió la metodología tradicional del productor de café, se usaron semillas de buena calidad seleccionadas In situ. Las semillas fueron pre-germinadas en camas germinadoras rústicas durante 45 días (punto de fosforo). Las plantitas más vigorosas fueron repicadas en bolsas almacigueras de $1 \mathrm{Kg}$, dos plantitas por bolsa, dejándolas en crecimiento bajo condiciones de tinglado hasta la emisión de cuatro pares de hojas ( 3 meses). El sustrato se consiguió al mezclar dos partes de tierra agrícola y una de compost de pulpa de café (2:1).

La siembra a campo definitivo se realizó ( 28 de febrero, 2013) en una parcela de café en proceso de renovación, iniciándose con el desmalezado y eliminación de plantas viejas. Los puntos de siembra fueron ubicados en curvas a nivel con distanciamiento de $1 \mathrm{~m}$ entre plantas y $2 \mathrm{~m}$ entre calles. Los hoyos, de $30 \times 30 \times 30 \mathrm{~cm}$, fueron cavados con ayuda de una cavadora recta. El mantenimiento posterior de la parcela experimental consistió en la eliminación de malezas con intervalos de un mes durante los primeros cuatro meses y después cada dos meses.

Todos los tratamientos recibieron cuatro aplicaciones del fungicida sistémico Tebunconazole + Triadimenol (Folicur Combi 300EC), a una dosis de $0,75 \%$, más el adherente Fosfatidilcolina ácido propionico Alkyl éter polioxietileno (LI 700), a una dosis de 1,5\%o, para el control de la roya amarilla. La primera aplicación se realizó a los dos meses y medio después de la siembra, la segunda a los tres meses y medio, la tercera a los cuatro meses y medio y la cuarta a los ocho meses y medio después de la siembra, con ayuda de una asperjadora manual de 20 litros.

\section{Muestreo de suelo para caracterización física y química}

Se tomaron muestras del suelo antes de la siembra de las plántulas de café en campo definitivo. El área del ensayo fue dividida en tres bloques y se colecto una muestra por bloque, cada muestra proveniente de $12 \mathrm{sub}$ muestras. Para el muestreo de suelos se siguió el procedimiento sugerido por Loli y Aquino (2011). Las muestras para su caracterización físico-química fueron enviadas al Laboratorio del Instituto de Cultivos Tropicales.

\section{Severidad de roya amarilla}

Se registró el grado de daño de la roya amarilla durante los doce meses correspondientes, utilizando la escala desarrollada por Sagarpa (2013) citado por Julca et al (2013) (Tabla 2). Con los datos obtenidos se calculó el porcentaje del índice de intensidad de daño (\%IID), donde \%IID es producto de la sumatoria del número de grado por el número de hojas con cada grado, entre número de grados mayor por número de hojas evaluadas.

Con los \%IID de cada evaluación se determinó la curva de progreso de la enfermedad y para graficarla se utilizó el \%IID promedio de cada tratamiento. Para el cálculo del área, bajo la curva de progreso de 
la enfermedad o AUDPC (Area Under Disease Progress Curve), se utilizó el \%IID así como la formula detallada más abajo (Campbell y Madden, 1990; Madden et al, 2007). Con los datos registrados del AUDPC se realizó el análisis de variancia y la prueba de Duncan para la comparación múltiple de medias $(\mathrm{P}=0,05)$, utilizando el programa estadístico Infostat/Profesional Versión 1.1.

$$
\text { AUDPC }=\sum_{i=1}^{N-1}\left(\frac{\mathrm{Yi}+\mathrm{Yi}+1}{2}\right)\left(\mathrm{T}_{i+1}-\mathrm{T}_{i}\right)
$$

Dónde: $\mathrm{Y}_{\mathrm{i}}$ es la proporción de tejido afectado en la observación i; $\left(T_{i+1}-T_{i}\right)$ el tiempo entre dos lecturas, $\mathrm{N}$ el número total de observaciones.

\section{RESULTADOS}

\section{Propiedades físicas y químicas del suelo}

De acuerdo con los resultados obtenidos del análisis previo de los suelos (Tabla 3), el estudio se realizó en un suelo fuertemente ácido, de textura arcillosa, con contenido medio de materia orgánica, el mismo nivel de $\mathrm{N}$ para las plantas y niveles deficientes a extremadamente deficientes de fósforo. La CIC es alta, dominada principalmente por el Calcio, con concentraciones altas de Magnesio y medias de Potasio. La mitad del área de estudio mostró un alto porcentaje de saturación de aluminio, mientras que la otra mitad del área presentó alta saturación de bases. Se estableció un fuerte desbalance catiónico, sobre todo entre el Ca con el K y el Mg con el K.

\section{Curva de índice de intensidad de daño}

Durante los doce meses de evaluación del porcentaje del índice de intensidad de daño (\%IID) de la roya amarilla, las curvas generadas muestran una tendencia descendente en todos los tratamientos, sin importar la variedad ni las dosis de nitrógeno.
Solamente en el segundo mes se registraron los más altos \%IID, pero fue mayor en la variedad Borbón que en la Caturra, alcanzando hasta el 57,50\%.

Las curvas del tratamiento control en ambas variedades mantuvieron tendencias crecientes hasta cinco meses después de la siembra, disminuyendo gradualmente de manera posterior. La variedad Borbón registró en octubre el \%IID más bajo $(17,63 \%)$, ocho meses después de la siembra, después la curva mostro tendencia creciente. La variedad Caturra registró el \%IID más bajo $(19,67 \%)$, nueve meses después de la siembra, siendo la tendencia posterior de la curva similar a la de la variedad Borbón (Figura 1).

Las curvas generadas en los tratamientos que recibieron abonamiento tuvieron comportamiento uniforme sin diferencias entre ellas. Según los cuatro últimos registros del \% IID, las curvas de los tratamientos con Caturra y Borbón muestran tendencia ascendente (Figura 2).

\section{Área bajo la curva de progreso de la enfermedad o AUDPC}

El cuadro de análisis de Variancia a un nivel de confianza de 0,05 para AUDPC, indica que no hay efecto significativo de la variedad sobre él \%IID de la roya amarilla, pero los tratamientos si muestran efecto significativo sobre su \%IID (Tabla 4).

Se obtuvieron valores de AUDPC más altos en el tratamiento control para ambas variedades. Las dosis de $\mathrm{N}$ no mostraron influencia significativa sobre él \%IID de la roya amarilla (Figura 3) durante los primeros doce meses, después de instalar las plántulas de café a campo definitivo, bajo condiciones de altura en la zona alta de Alonzo de Alvarado Roque. En términos generales, la variedad Borbón registra mayores valores de AUDPC, sugiriendo que esta variedad muestra un mejor comportamiento a los tratamientos de abono nitrogenado.

Tabla 1. Dosis de Nitrógeno evaluado para determinar la respuesta sobre la severidad de daño de la RA en las variedades Caturra y Borbón.

\begin{tabular}{cccccc}
\hline \multirow{2}{*}{ TRAT } & \multicolumn{5}{c}{ Dosis aplicadas $\left(\mathrm{K} \mathrm{gha}^{-1}\right)$} \\
\cline { 2 - 6 } & $\mathbf{N}$ & $\mathbf{P}$ & $\mathbf{K}$ & $\mathbf{M g}$ & $\mathbf{S}$ \\
\hline 1 & 50 & 80 & 150 & 88 & 60 \\
2 & 75 & 80 & 150 & 88 & 60 \\
3 & 100 & 80 & 150 & 88 & 60 \\
4 & 125 & 80 & 150 & 88 & 60 \\
5 & 0 & 0 & 0 & 0 & 0 \\
\hline
\end{tabular}


Tabla 2. Escala descriptiva para evaluar la severidad roya en campo del café (Hemileia vastatrix) en el campo (Sagarpa, 2013).

\begin{tabular}{cl}
\hline Grado & \multicolumn{1}{c}{ Descripción } \\
\hline 0 & Sano o sin síntomas visibles \\
1 & Síntomas visibles llegando de 1 a $5 \%$ del área total sana \\
2 & Las manchas empiezan a unirse llegando a ocupar del 6 al $20 \%$ del área sana \\
3 & Las hojas comienzan a necrosarse de manera muy notoria afectando el 21 al $50 \%$ del área sana \\
4 & Mayor al $50 \%$ del área foliar se encuentra afectada \\
\hline
\end{tabular}

Tabla 3. Propiedades físicas y químicas de los suelos de los experimento para determinar la respuesta de dos variedades de $C$. arabica al abonamiento nitrogenado con relación a la severidad de daño de la roya amarilla.

\begin{tabular}{|c|c|c|c|c|c|c|c|c|c|c|c|c|c|c|c|c|c|}
\hline \multirow{3}{*}{ Variedad } & \multirow{3}{*}{ Bloque $\mathrm{pH}$} & \multirow{3}{*}{$\begin{array}{c}\text { C.E } \\
\mathrm{dS} / \mathrm{m}\end{array}$} & \multirow{3}{*}{$\begin{array}{c}\mathrm{CaCO}_{3} \\
(\%)^{3}\end{array}$} & \multirow{3}{*}{$\begin{array}{l}\text { M.0 } \\
(\%)\end{array}$} & \multirow{3}{*}{$\begin{array}{c}\mathbf{N} \\
(\%)\end{array}$} & \multirow{3}{*}{$\underset{(\mathrm{ppm})}{\mathrm{P}}$} & \multirow{3}{*}{$\begin{array}{c}\mathrm{K} \\
(\mathrm{ppm})\end{array}$} & \multicolumn{4}{|c|}{ ANALISIS MECANICO } & \multirow{2}{*}{\begin{tabular}{|c|c|} 
C.I.C. \\
efectiva
\end{tabular}} & \multicolumn{3}{|c|}{ CATIONES CAMBIABLES } & \multirow{3}{*}{$\begin{array}{l}\text { Suma } \\
\text { de } \\
\text { bases }\end{array}$} & \multirow{3}{*}{$\begin{array}{c}\% \text { Sat. } \\
\text { de } \\
\text { bases }\end{array}$} \\
\hline & & & & & & & & \multirow{2}{*}{\multicolumn{2}{|c|}{\begin{tabular}{|c|} 
Arena Limo \\
$\%$ \\
\end{tabular}}} & \multirow{2}{*}{ Arcilla } & \multirow{2}{*}{$\begin{array}{c}\text { CLASE } \\
\text { CULTURAL }\end{array}$} & & $\mathrm{Ca}^{2+} \mathrm{Mg}^{2+}$ & $\mathrm{K}^{+}$ & $\mathrm{Na}^{+} \mathrm{Al}^{+4}+\mathrm{H}^{+}$ & & \\
\hline & & & & & & & & & & & & & $\mathrm{meq} / 100$ & & & & \\
\hline & BI 3.85 & 0.08 & 0.00 & 2.60 & 0.12 & 7.03 & 44 & 18.24 & 28.28 & 53.48 & Arc & 16.64 & $3.46 \quad 0.66$ & 0.11 & 0.0012 .41 & 4.23 & 25.41 \\
\hline \multirow[t]{3}{*}{ Caturra } & B II 4.31 & 0.05 & 0.00 & 2.33 & 0.11 & 6.01 & 61 & 16.24 & 28.28 & 55.48 & Arc & 16.88 & 11.991 .81 & 0.16 & $0.00 \quad 2.93$ & 13.95 & 82.66 \\
\hline & B III 4.05 & 0.05 & 0.00 & 1.89 & 0.08 & 2.93 & 52 & 18.24 & 26.28 & 55.48 & $\operatorname{Arc}$ & 14.78 & $5.77 \quad 0.90$ & 0.13 & 0.007 .97 & 6.81 & 46.06 \\
\hline & B I 4.61 & 0.11 & 0.00 & 2.45 & 0.11 & 6.01 & 59 & 18.24 & 30.28 & 51.48 & Arc & 20.78 & 6.691 .42 & 0.15 & 0.0012 .51 & 8.27 & 39.78 \\
\hline \multirow[t]{2}{*}{ Borbón } & B II 4.52 & 0.07 & 0.00 & 2.81 & 0.13 & 3.08 & 55 & 20.24 & 24.28 & 55.48 & $\operatorname{Arc}$ & 10.59 & 6.491 .64 & 0.14 & 0.002 .32 & 8.26 & 78.08 \\
\hline & B III 4.63 & 0.07 & 0.00 & 2.49 & 0.11 & 4.83 & 47 & 16.24 & 24.28 & 59.48 & Arc & 15.41 & 12.671 .81 & 0.12 & $0.00 \quad 0.81$ & 14.60 & 94.76 \\
\hline
\end{tabular}

Tabla 4. Análisis de variancia del área bajo la curva de progreso de la enfermedad o AUDPC $(p=0.05)$.

\begin{tabular}{lccccc}
\multicolumn{1}{c}{ F.V. } & SC & GI & CM & F & p-valor \\
\hline Variedad & 546750 & 1 & 546750 & 1.16 & 0.2953 \\
Tratamiento & 35940168.8 & 4 & 8985042.19 & 19.09 & $<0.0001$ \\
Bloque & 449328.75 & 2 & 224664.37 & 0.48 & 0.628 \\
Variedad*Tratamiento & 2163956.25 & 4 & 540989.06 & 1.15 & 0.3653 \\
Error & 8469896.25 & 18 & 470549.79 & & \\
Total & 47570100 & 29 & & & \\
\hline
\end{tabular}



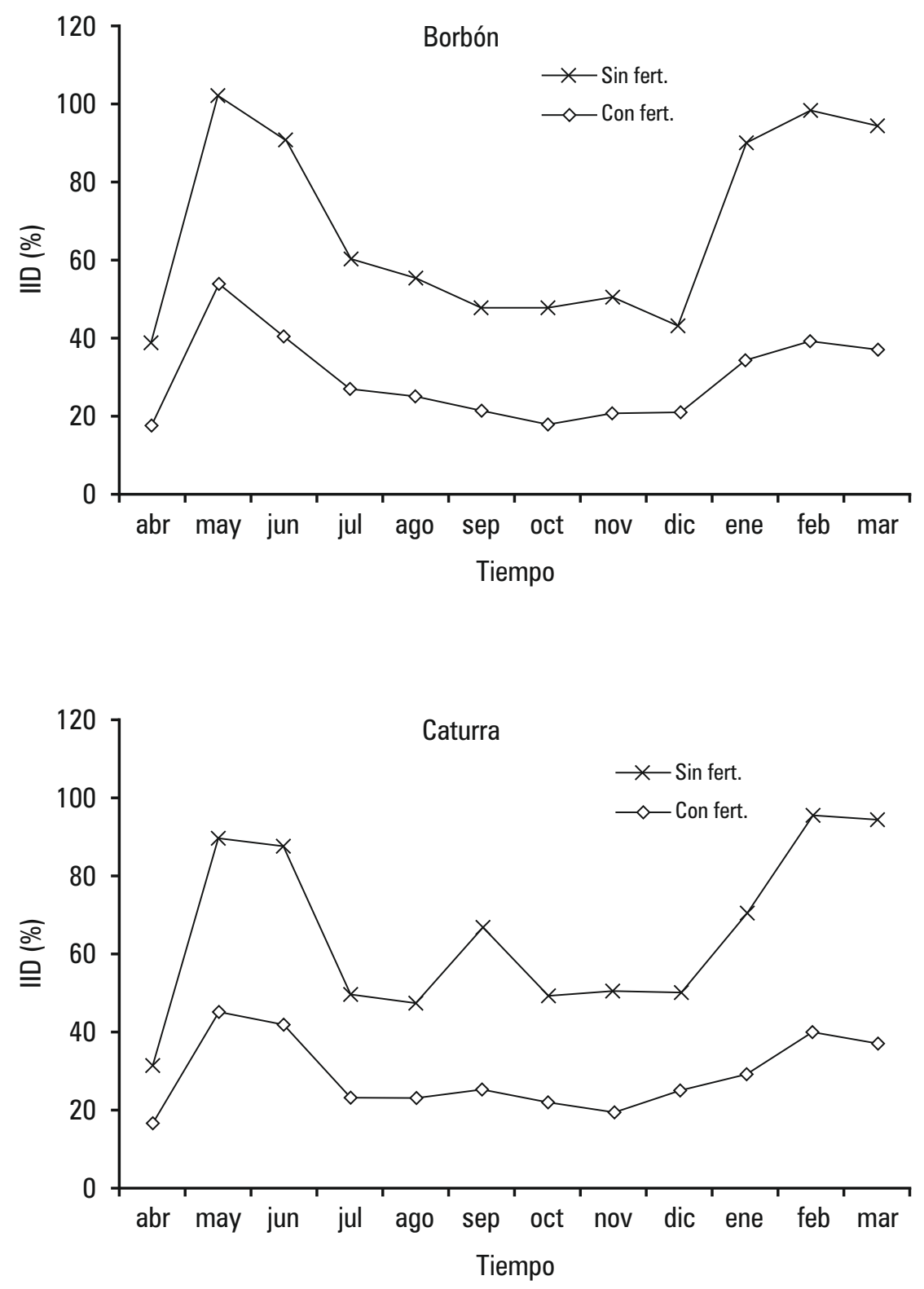

Figura 1. Curva de progreso del \%llD de la roya amarilla en dos variedades de $C$. arabica Caturra y Borbón, con el abonamiento nitrogenado y sin fertilización. 

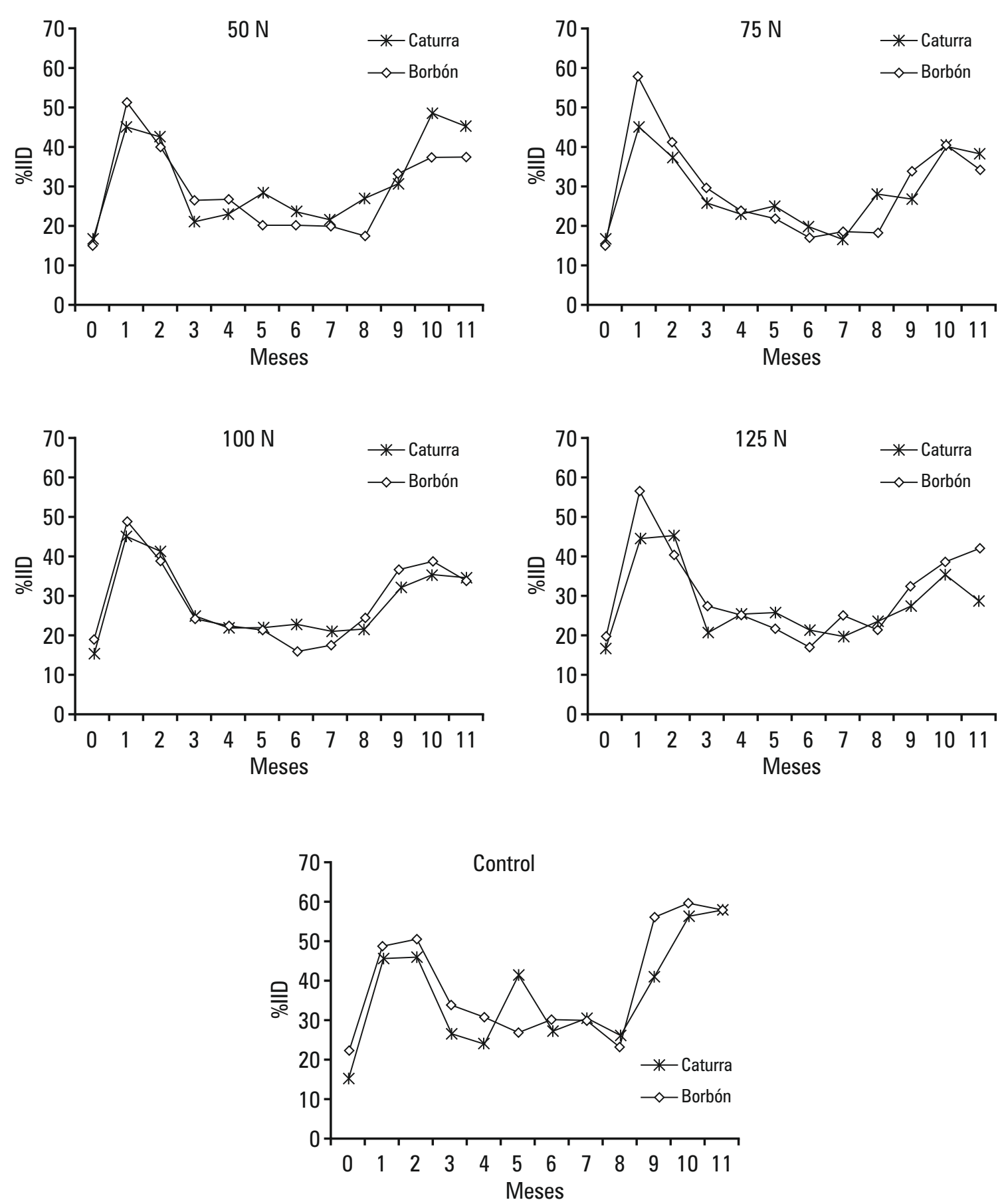

Figura 2. Curva de progreso del \%IID de la roya amarilla en dos variedades de C. arabica Caturra y Borbón, influenciados por las dosis de nitrógeno. 


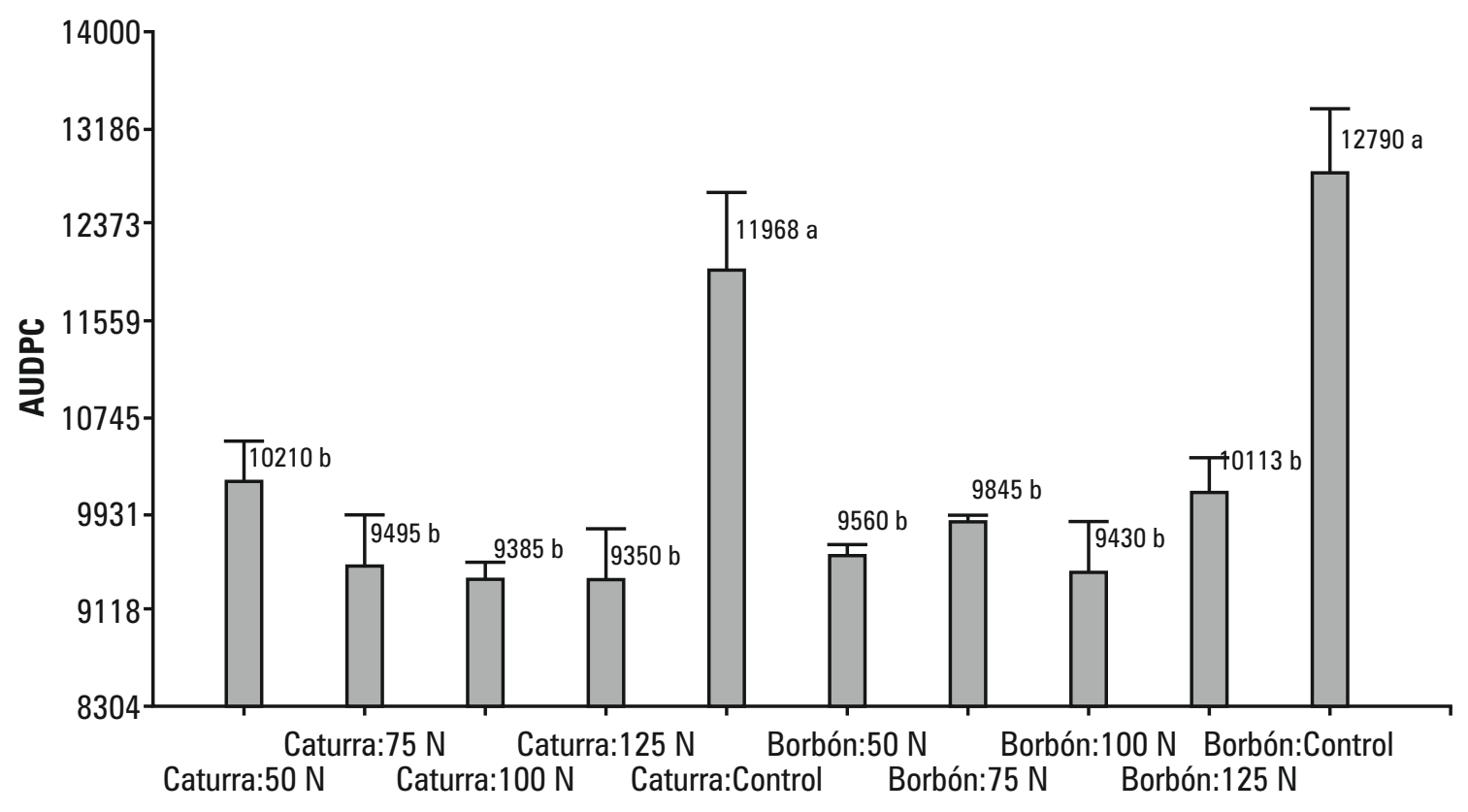

$$
R^{2}=0.82 \quad C V=6.72
$$

Variedad*Dosis N

Figura 3. AUDPC de la roya amarilla en dos variedades de $C$. arabica bajo condiciones de zona alta de Alonzo de Alvarado Roque. Las barras con letras distintas indican diferencias estadísticas significativas según la prueba de Duncan $(p<=0.05)$. 


\section{DISCUSIONES}

Las plantas en general requieren de un sistema de nutrición balanceada, ya que su productividad es afectada por los factores de carencia o déficit nutricional, así como por las propiedades físicas del suelo (Vera, 2004; INPOFOS, 1997; Baligar, et al, 1990). El estudio fue realizado sobre un suelo pesado que retiene alta humedad en las épocas de lluvia y sufre desecamiento en las épocas secas, afectando el adecuado desplazamiento de los nutrientes del suelo hasta las raíces de las plantas de café.

El fuerte desbalance catiónico establecido en el ensayo, sobre todo entre el $\mathrm{Ca}$ con el $\mathrm{K}$ y el $\mathrm{Mg}$ con el $\mathrm{K}$, sugiere que las plantas de café tuvieron una mínima absorción de estos nutrientes, tal como lo sugieren Suárez et al (2010); Fageria et al (2002); Bertsch (2003), INPOFOS (1997), ya que la alta concentración de Calcio en el suelo inhibe la absorción de K y Mg para la gran mayoría de plantas. Los altos contenidos de calcio y magnesio probablemente estén asociados al reciclaje de nutrientes por los residuos orgánicos generados por las plantas de sombra (Inga $s p$ ) y del café mismo, a través de los años de cultivo del área en estudio (Szott et al, 1991, Alegre y Rao, 1996). Trabajos realizados por Mutter (1998), Chinchilla (2004) e INPOFOS (1997), citado por Suárez et al (2010), indican que plantas con deficiencias de $\mathrm{K}$ y $\mathrm{Mg}$, son susceptibles a plagas y enfermedades, siendo las plantas deficientes en $\mathrm{K}$ mucho más susceptibles a estos ataques.

Durante los doce meses de evaluación del \%IID de la roya amarilla, el comportamiento de la enfermedad presentó una fase de desarrollo lento de julio a diciembre, luego se incrementó de forma rápida a partir de diciembre a enero. La curva de la enfermedad alcanzó su punto máximo entre febrero y mayo del siguiente año, comportamiento similar a lo encontrado por Orozco et al, (2011) en Guatemala y por Méndez (1982) citado por Rivillas et al, (2011) en México. Se registraron daños más severos de la roya amarilla entre diciembre y mayo, posiblemente influenciados por la coincidencia con los meses de mayor precipitación en la región, coincidiendo con Rivillas et al, (2011), quien menciona que el mayor incremento de la enfermedad se observa en los meses de mayor precipitación. Para las plantaciones en producción los daños también son severos durante los meses de desarrollo y maduración del fruto (López, 2010), en la época de cosecha.

La dosis de $\mathrm{N}$ no influyeron en la severidad de daño de la roya amarilla durante los doce meses de evaluación en ambas variedades. Carvalho et al, (2006), al respecto, menciona que el requerimiento de $\mathrm{N}$ se incrementa con la edad de la planta, especialmente a inicios de la producción de granos, (Costa et al., 2006). El experimento fue realizado en un suelo con contenido medio de $\mathrm{N}$ para las plantas, posiblemente las plantas durante su primer año de desarrollo utilizaron solo el $\mathrm{N}$ disponible del suelo $(0.11 \mathrm{ppm})$. El incremento del número de pares de hojas y ramas plagiotrópicas, son respuestas a un suministro adecuado de Nitrógeno (Nazareno et al, 2003). Su exceso induce el incremento de la concentración de aminoácidos y amidas en la planta, que además de provocar alteraciones en las relaciones $\mathrm{N} / \mathrm{P}$ y $\mathrm{N} / \mathrm{K}$, también participa en la constitución de aminoácidos, proteínas, enzimas, ácidos nucleicos, pigmentos, hormonas de crecimiento, fitoalexinas y fenoles (Costa et al., 2006). Agrios, (2005) menciona a las fitoalexinas como una sustancia anti fúngica.

Los valores de AUDPC obtenidos en los tratamientos control (Caturra $=11968$ y Borbón $=$ 12790), en comparación a los tratamientos con abonamiento indistintamente de la dosis de Nitrógeno, evidencian que las condiciones fueron favorables para el desarrollo de la enfermedad, tal como menciona Márquez-Dávila, (2007). Los resultados indican que el abonamiento influyó significativamente sobre él \%IID de la roya amarilla, tal como mencionan Alarcón et al (1996) y Da Silva et al (2009). En términos generales, la variedad Borbón registra mayores valores de AUDPC, sugiriendo los datos que esta variedad muestra mayor susceptibilidad a la roya amarilla que la variedad Caturra.

\section{CONCLUSIONES}

No hubo efecto significativo ni de la variedad ni de las dosis de Nitrógeno sobre la severidad de daño de la roya amarilla (Hemileia vastatrix Berck et. $B r$.). El abonamiento influyó significativamente sobre la severidad de daño de la roya amarilla en los estadios iniciales de café en condiciones de la zona alta Alonzo de Alvarado Roque.

\section{LITERATURA CITADA}

Agrios, G.N. 1997. Plant Pathology, 4th Ed. Academic Press, California, USA, 635 pp. Chapters 5 and 6.

Alarcón M,O.; Aldazabal, M.; Martínez, J. 1996. Influencia del sol y la sombra en la calidad y el rendimiento del grano de café. Centro Agrícola 23(3): 11-16.

Alegre, J.C.; Rao, M.R. 1996. Soil conservation by contour hedgerows of Inga edulis on slopes in 
the humid tropics of Peru. Agriculture, Ecosystems, Enviroment, 57: 17-25.

Baligar, V.C.; Duncan, R.R.; Fageria, N.K. 1990. Soil-plant interaction on nutrient use efficiency in plantas: An overwiew. In: Baligar, V.C.; Duncan, R.R. (Eds). Crops as Enhancers of Nutrient Use. p. 351-373.

Bertsch, F. 2003. Absorción de nutrimentos por los cultivos. Asociación Costarricense de la Ciencia del Suelo, Costa Rica. 307pp.

Campbell, C.L.; Madden, L.V. 1990. Introduction to plant disease epidemiology. Wiley, New York, USA. 532 pp.

Catani, R.A.; Moraes, F.R.P. 1958. A composição química do cafeeiro. Revista da Agricultura, 33:45-52.

Carvalho, C.M.L.; Irineu, F.J.; and Cochicho, R. J.D. 2006. Aspects of nitrogen metabolism in coffee plants. Brazilian Journal of Plant Physiology, 18(1): 9-21. Doi: 10.1590/S167704202006000100002

Costa, M.J.N.; Zambolim, L.; Rodrigues, F.A. 2006. Efeito de níveis de desbaste de frutos do cafeeiro na incidência da ferrugem, no teor de nutrientes, carboidratos e açúcares redutores. Fitopatologia Brasileira, 31(6): 564-571. Doi: 10.1590/S0100-41582006000600005

Crop Protection Compendium (CABI). 2007. CAB Internationa 1 , Wallingford, UK (http://www.cabi.org). Acceso: 17/01/2010.

Da Silva, A.L.; Bruno, I.P.; Reichardt, K.; Bacchi, O.O.S.; Dourado-Neto, D.; Favarin, J.L.; Da Costa, F.M. P.; Timm, L.C. 2009. Soil water extraction by roots and $\mathrm{Kc}$ for the coffee crop. Revista Brasileira de Enghenaria Agrícola e Ambiental, 13(3): 257-261. doi: 10.1590/S1415-43662009000300006

Fageria, N.K.; Baligar, V.C.; Clark, R.B. 2002. Micronutrients in Crop Production. Advances in Agronomy, 77: 185-268. doi: 10.1016/S00652113(02)77015-6
Julca, O.A.; Echevarría, A.C.; Ladera, M.Y.; Borjas, V.R.; Cruz, J.R.; Bello, A.S., y Crespo, C.R. 2013. Una revisión sobre la roya del café (Hemileia vastatrix) algunas experiencias y recomendaciones para el Perú. Agroenfoque, 28: 28-31

Madden, L.V.; Hughes, G.; Van den Bosch, F. 2007. The Study of plant disease epidemics. The American Phytopathological Society. 421 pp.

Nazareno, R.B.; Oliveira C.A.S.; Sanzonowicz, C.; Sampaio, J.B.R.; Silva, J.C.P.; Guerra, A.F. 2003 Crescimento inicial do cafeeiro Rubi em resposta a doses de nitrogênio, fósforo e potássio e a regimes hídricos. Pesquisa Agropecuária Brasileira, 38(8) :903-910. doi: 10.1590/S0100204X2003000800002

Szott, L. T., Palm, C.A., Sanchez, P.A. 1991. Agroforestry in acid soils of the humid tropics. Advances in Agronomy, 45: 275-301. doi: 10.1016/S0065-2113(08)60043-7

Tirado, L.J. 2008. Enfermedades fungosas del cultivo de café. Primera edición. Consejo Nacional de Ciencia, Tecnología e Innovación Tecnológica-CONCYTEC. 183pp.

Suárez, A., M. Calvache, R. Jaramillo, R. Morales. 2010. Recuperación de palma aceitera (Elaeis guineensis jacq.) bajo estrés por desbalance catiónico de $\mathrm{Ca}, \mathrm{Mg}$ y $\mathrm{K}$ con el uso de diferentes fuentes de $\mathrm{Mg}$ y $\mathrm{K}$. [XII Congreso Ecuatoriano de la Ciencia del Suelo]. (http://www.secsuelo. org/XIICongreso/Simposios/Nutricion/Ponenci as $/ 2 . \% 20$ Andres $\% 20$ Suarez. $\% 20 \% 20$ ARTICU LO\%20RECUPERACION\%20DE\%20PALM AS.pdf) Acceso: 10/07/2013.

The American Phytopathological Society (APS). 2011. Coffee rust (Hemileia vastatrix). (http://www.apsnet.org/edcenter/intropp/lesson s/fungi/Basidiomycetes/Pages/CoffeeR ust.aspx.) Acceso: 20/08/2013.

Recibido: 20/01/2014

Aceptado para publicación: 22/03/2014 